\title{
WaveNet-Based Deep Neural Networks for the Characterization of Anomalous Diffusion (WADNet)
}

\author{
Dezhong Li, Qiujin Yao, Zihan Huang \\ School of Physics and Electronics, Hunan University, Changsha 410082, China \\ E-mail: huangzih@hnu.edu.cn
}

\begin{abstract}
Anomalous diffusion, which shows a deviation of transport dynamics from the framework of standard Brownian motion, is involved in the evolution of various physical, chemical, biological, and economic systems. The study of such random processes is of fundamental importance in unveiling the physical properties of random walkers and complex systems. However, classical methods to characterize anomalous diffusion are often disqualified for individual short trajectories, leading to the launch of the Anomalous Diffusion (AnDi) Challenge. This challenge aims at objectively assessing and comparing new approaches for single trajectory characterization, with respect to three different aspects: the inference of the anomalous diffusion exponent; the classification of the diffusion model; and the segmentation of trajectories. In this article, to address the inference and classification tasks in the challenge, we develop a WaveNet-based deep neural network (WADNet) by combining a modified WaveNet encoder with long short-term memory networks, without any prior knowledge of anomalous diffusion. As the performance of our model has surpassed the current 1st places in the challenge leaderboard on both two tasks for all dimensions (6 subtasks), WADNet could be the part of state-of-the-art techniques to decode the AnDi database. Our method presents a benchmark for future research, and could accelerate the development of a versatile tool for the characterization of anomalous diffusion.
\end{abstract}

Keywords: anomalous diffusion, single trajectory characterization, anomalous diffusion challenge, machine learning, deep neural network, WaveNet 


\section{Introduction}

A random walker undergoes anomalous diffusion when its mean squared displacement (MSD) deviates from a linear temporal evolution [1,2]. Close attention is paid to such diffusion process in a variety of scientific fields, such as physics [3 6], chemistry [7], biology [8 12], economics [13 15], and social science [16]. The raw information of anomalous diffusion dynamics can be recorded as trajectories of random walkers via techniques like single particle tracking $17-21$. Since these walkers directly probe their surroundings [22 27], an in-depth characterization of their trajectory data is of significant importance in not only understanding the underlying mechanisms of anomalous diffusion, but also uncovering the intrinsic properties of both random walkers and complex environments.

Classical methods to analyze these trajectories are mostly based on mathematical statistics. A representative example is the calculation of MSD, which is quantified by the time-average or ensemble-average of squared displacements during a time interval 28, 29]. These approaches can be valid to detect the diffusion properties when the trajectory is sufficiently long or a quantity of trajectories is available. However, as a consequence of the limitations of experimental techniques, one can usually obtain few, short, and noisy trajectories when recording a diffusion process $[30-32]$. These trajectories suffer the undesirable variations introduced by noise and a lack of significant statistics. Thus, mining information of anomalous diffusion from such a dataset are rather difficult and challenging for standard statistics-based approaches.

Therefore, alternative methods with improved performance on the characterization of anomalous diffusion are in great demand. This urgent issue leads to the launch of the Anomalous Diffusion (AnDi) Challenge (http://www.andi-challenge.org) which is spearheaded by a team of notable scientists [33]. The AnDi Challenge aims at assessing and comparing new approaches that go beyond classical methods for single trajectory characterization, regarding three tasks: the inference of the anomalous diffusion exponent; the classification of the diffusion model; and the segmentation of trajectories. Each task is split into 3 subtasks to deal with trajectories in different spatial dimensions (1D, 2D, and 3D). A challenge leaderboard is available by scoring the predictions of participating teams on a common test dataset. The rank of a team in the leaderboard can be an intuitive and objective criterion for the performance of corresponding method.

On the other hand, an unprecedented revolution of machine learning technologies has been witnessed in the past few years 34 39]. In particular, deep learning models that process sequential (or time-series) data are recently undergoing a rapid development 36 39], from traditional recurrent neural networks (RNNs) 36, 37 to the attentionbased Transformer [39]. These models can automatically learn the rules to extract useful information from sequences without any prior knowledge. Since trajectories of random walkers are actually sequences, these deep networks are highly expected to be qualified for the characterization of anomalous diffusion $[40-46]$. In this article, as a response 

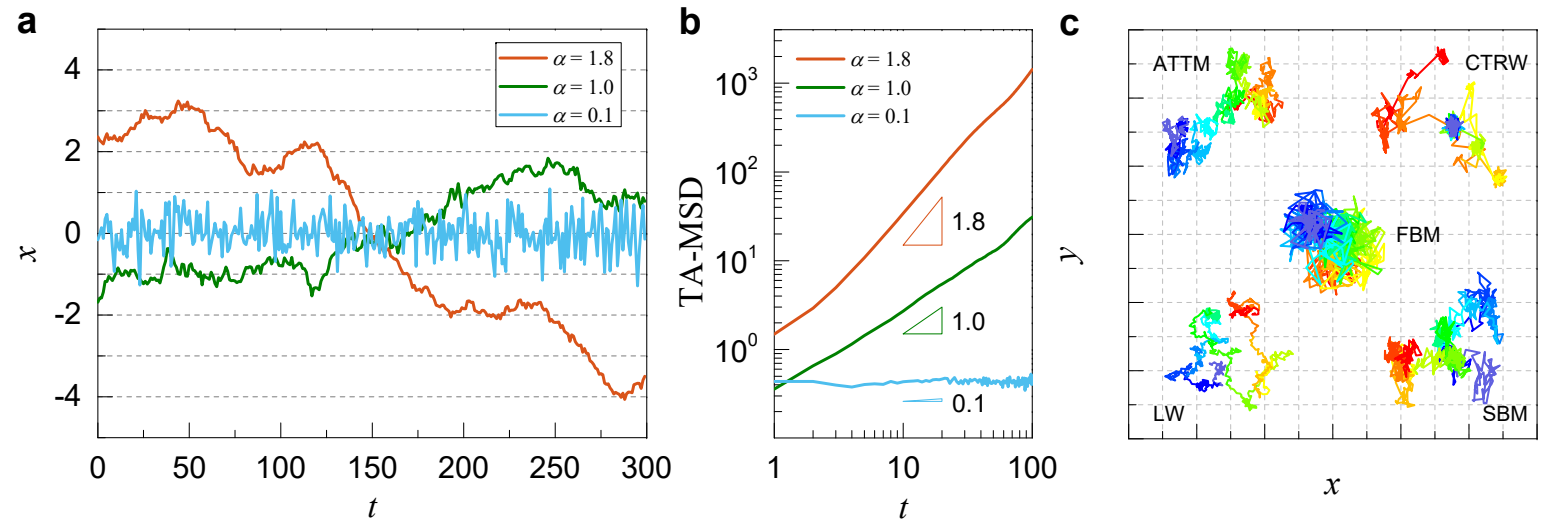

Figure 1. Schematic illustrations of the inference and classification tasks in the AnDi Challenge. (a) Representative 1D trajectories (part) of subdiffusion $(\alpha=0.1)$, Brownian diffusion $(\alpha=1.0)$, and superdiffusion $(\alpha=1.8)$, respectively. (b) Time evolutions of TA-MSD corresponding to three trajectories in (a). The exponent $\alpha$ can be determined by fitting the slope (shown as the triangle) of the log-log plot of TAMSD versus time. (c) Representative trajectories of five diffusion models in the AnDi database: annealed transient time motion (ATTM), continuous-time random walk (CTRW), fractional Brownian motion (FBM), Lévy walk (LW), and scaled Brownian motion (SBM). The color on the trajectory denotes the time.

to the AnDi Challenge, we develop a WaveNet-based deep neural network (WADNet) by combining a modified WaveNet encoder [38] with long short-term memory (LSTM) networks [36], to address two tasks in the challenge: the inference of the anomalous diffusion exponent, and the classification of the diffusion model. Despite that no prior knowledge of anomalous diffusion is provided for WADNet, the performance of our model has surpassed the current 1st places in the challenge leaderboard on both two tasks for all dimensions (6 subtasks). Such a result suggests that WADNet could be the part of state-of-the-art techniques to decode the AnDi database.

\section{Methods}

\subsection{Tasks, evaluation metrics, and datasets}

In this work, we focus on two tasks in AnDi Challenge: the inference of the anomalous diffusion exponent (the inference task), and the classification of the diffusion model (the classification task). Brief introductions to these two tasks and corresponding evaluation metrics are given as follows:

- Inference of the anomalous diffusion exponent. One of the most common behaviors in anomalous diffusion dynamics is the nonlinear growth of MSD versus time, given by:

$$
\operatorname{MSD}(t) \sim t^{\alpha} .
$$

Here, the anomalous diffusion exponent $\alpha$ is a real positive number. For standard Brownian diffusion we have $\alpha=1$, while for superdiffusion (subdiffusion) we have 
$\alpha>1(\alpha<1)$. Representative 1D trajectories (part) of these three diffusion patterns are demonstrated in Figure 1a where $\alpha=0.1,1.0$, and 1.8 respectively. To identify the exponent $\alpha$ of a single trajectory $\mathbf{x}(t)$, traditional statistics-based method is calculating the time-averaged mean squared displacement (TA-MSD), written as 41]:

$$
\operatorname{TA}-\operatorname{MSD}(t)=\frac{1}{t_{\max }-t} \int_{0}^{t_{\max }-t}\|\mathbf{x}(\tau+t)-\mathbf{x}(\tau)\|_{2}^{2} \mathrm{~d} \tau
$$

For example, time evolutions of TA-MSD corresponding to three trajectories in Figure 1a are given in Figure 1b. As shown by the triangles, the exponent $\alpha$ can be determined by fitting the slope of the log-log plot of TA-MSD versus time. However, such an approach relies heavily on a sufficient long trajectory, and the result can usually be sensitive to the manual selection of fitting range. Hence, in this task, AnDi Challenge calls for improved methods to infer the anomalous diffusion exponent of a single short trajectory. The evaluation metric for this regression problem is the mean absolute error (MAE):

$$
\mathrm{MAE}=\frac{1}{N} \sum_{i=1}^{N}\left|\alpha_{i, \mathrm{p}}-\alpha_{i, \mathrm{GT}}\right|
$$

where $N$ is the number of trajectories in the test dataset, $\alpha_{i, \mathrm{p}}$ and $\alpha_{i, \mathrm{GT}}$ are the predicted and ground truth values of anomalous diffusion exponent of the $i$-th trajectory, respectively.

- Classification of the diffusion model. It is well known that the properties of standard Brownian motion can be captured by the Wiener process. Analogously, the dynamics of anomalous diffusion can also be described by various theoretical stochastic process models. However, confident identification of these models from trajectory data is quite challenging for classical methods. Thus, to address this issue, the participants in this task are requested to develop valid approaches to classify the diffusion model of a single short trajectory. Five typical diffusion models are included in the challenge. Here, we give a brief introduction to these models and show the range of corresponding $\alpha$ in the AnDi database:

- Annealed transient time motion (ATTM) [47], a non-ergodic model that describes a Brownian motion with the diffusion coefficient randomly varying either in time or space $(0.05 \leq \alpha \leq 1)$.

- Continuous-time random walk (CTRW) [48], a non-ergodic model where the waiting time of a random walker between two subsequent steps is irregular and randomly chosen $(0.05 \leq \alpha \leq 1)$.

- Fractional Brownian motion (FBM) [49], an ergodic diffusion process that is driven by a fractional Gaussian noise. Such noise is normal distributed but has a power-law correlation $(0.05 \leq \alpha<2)$.

- Lévy walk (LW) [50|, a non-ergodic model where the waiting time between subsequent steps is also irregular as CTRW but the step length is not Gaussian distributed $(1 \leq \alpha \leq 2)$. 
Table 1. List of specific lengths and corresponding trajectory numbers in training set.

\begin{tabular}{cccccc}
\hline Length & Number & Length & Number & Length & Number \\
\hline 10 & 5000000 & 105 & 2000000 & 400 & 1000000 \\
15 & 5000000 & 115 & 2000000 & 425 & 1000000 \\
20 & 5000000 & 120 & 2000000 & 450 & 1000000 \\
25 & 5000000 & 125 & 2000000 & 475 & 1000000 \\
30 & 5000000 & 150 & 2000000 & 500 & 1000000 \\
40 & 5000000 & 175 & 2000000 & 550 & 1000000 \\
45 & 5000000 & 200 & 2000000 & 600 & 1000000 \\
50 & 5000000 & 225 & 2000000 & 650 & 1000000 \\
55 & 5000000 & 250 & 2000000 & 700 & 1000000 \\
60 & 5000000 & 250 & 2000000 & 750 & 1000000 \\
70 & 5000000 & 275 & 1500000 & 800 & 1000000 \\
80 & 5000000 & 300 & 1500000 & 850 & 1000000 \\
90 & 5000000 & 325 & 1500000 & 900 & 1000000 \\
100 & 5000000 & 350 & 1500000 & 950 & 1000000 \\
105 & 2000000 & 375 & 1500000 & & \\
\hline
\end{tabular}

- Scaled Brownian motion (SBM) [51], a non-ergodic model that describes a Brownian motion with a deterministically time-dependent diffusion coefficient $(0.05 \leq \alpha \leq 2)$.

Detailed descriptions regarding the mechanisms of these theoretical models can be found in the instruction of AnDi Challenge [33]. Representative trajectories of these five models are shown in Figure 1c, where color on the trajectory denotes the time. The evaluation metric in this classification task is the "micro" version of F1-score, expressed as:

$$
\mathrm{F} 1=2 \cdot \frac{\text { precision } \cdot \text { recall }}{\text { precision }+ \text { recall }}
$$

Here, precision $=\frac{\mathrm{TP}}{\mathrm{TP}+\mathrm{FP}}$ and recall $=\frac{\mathrm{TP}}{\mathrm{TP}+\mathrm{FN}}$, where $\mathrm{TP}, \mathrm{FP}$ and $\mathrm{TN}$ represent the total number of true positives, false positives and false negatives in the classification result, respectively.

The datasets in the AnDi Challenge are composed of simulated trajectories that are generated by an open-source Python package andi-dataset (https://github.com/ AnDiChallenge/ANDI_datasets [52. The working principle of andi-dataset is briefly summarized here. Trajectory generation is based on the theoretical framework of five diffusion models in the database, where time steps are uniformly sampled with a unitary time interval. The ground truth value of anomalous diffusion exponent $\alpha_{\mathrm{GT}}$ ranges from 0.05 to 2.0 with a step 0.05 . Trajectory length varies from 10 to 999 points, where one point denotes the spatial coordinate of the random walker at one time step. In particular, to mimic real experimental data, simulated trajectories are corrupted with zero-mean Gaussian noise. The signal-to-noise ratio (SNR) of a trajectory can be regulated through 
changing the standard deviation $\sigma_{n}$ of noise distribution $\left(\mathrm{SNR}=1 / \sigma_{n}\right)$. In the andidataset, $\sigma_{n}$ is set as 1.0, 0.5, or 0.1, resulting in $\mathrm{SNR}=1,2$, or 10 .

On the basis of andi-dataset, a test dataset consisting of 10000 trajectories is generated by organizers as the common dataset for leaderboard scoring. The training dataset we utilized in this work is also generated by the package andi-dataset. For both the inference and classification tasks, we choose 43 specific lengths and separately generate the training subsets at these lengths. The values of these specific lengths and corresponding trajectory numbers are listed in Table 1. Before training or inference, trajectory data is normalized to ensure its position's average and standard deviation in each dimension are 0 and 1 respectively.

\subsection{Workflow of WADNet}

The overview of the entire workflow of WADNet is demonstrated in Figure 2, including three main parts: Input, WaveNet Encoder, and RNN \& MLP. As shown in the Input part, input data can be obtained through a simple preprocessing of the raw trajectory, which is valid for trajectories in arbitrary dimensions: i. we separate the trajectory in each dimension into 1D time-series data; ii. we stack the 1D time-series data of all dimensions to compose a multi-channel tensor as the input of WADNet. For instance, the transformation of a $3 \mathrm{D}$ trajectory $\mathbf{x}(t)=\left[x_{1}(t), x_{2}(t), x_{3}(t)\right]^{\mathrm{T}}$ with a length $T$ to an input tensor $\overline{\mathbf{x}}$ can be expressed as:

$$
\left\{\left[\begin{array}{l}
x_{1}(1) \\
x_{2}(1) \\
x_{3}(1)
\end{array}\right],\left[\begin{array}{l}
x_{1}(2) \\
x_{2}(2) \\
x_{3}(2)
\end{array}\right], \cdots,\left[\begin{array}{l}
x_{1}(T) \\
x_{2}(T) \\
x_{3}(T)
\end{array}\right]\right\} \rightarrow\left\{\begin{array}{l}
{\left[x_{1}(1), x_{1}(2), \cdots, x_{1}(T)\right]} \\
{\left[x_{2}(1), x_{2}(2), \cdots, x_{2}(T)\right]} \\
{\left[x_{3}(1), x_{3}(2), \cdots, x_{3}(T)\right]}
\end{array}\right\}
$$

After the preprocessing, the input tensor is going to pass through a modified WaveNet encoder. WaveNet is a deep neural network designed by Google Deepmind for the purpose of raw audio generation [38]. The encoder of WaveNet based on dilated causal convolutions, gated activation units, and skip connections has been proved to be a powerful tool for extracting information from sequential data [53,54. In the WaveNet Encoder part of Figure 2, we show the detailed architecture of the modified WaveNet encoder utilized in this work. The input tensor $\overline{\mathbf{x}}$ is firstly filtered by a convolutional layer with filter size $1 \times 3$ and filter number 32 . Corresponding output $\tilde{\mathbf{x}}_{1}$ is subsequently processed by the stacked dilated convolutions with a dilation depth $d=16$. Here, a dilated convolution with a dilation $m$ is a convolution operation where the filter skips the input values with a step $m-1$. Such a structure allows the filter to deal with a larger area than its size. For example, a $3 \times 3$ filter with a dilation 4 can be directly applied on a $9 \times 9$ area. Thus, by simply stacking the dilated convolutions, networks are enabled to have very large receptive fields with just a few layers. In this work, the dilation is doubled for every layer, which is the same as the original WaveNet 38.

Next, to apply the gated activation unit in each layer, dilated convolution in the WaveNet encoder is composed of two parallel convolution operators with same 

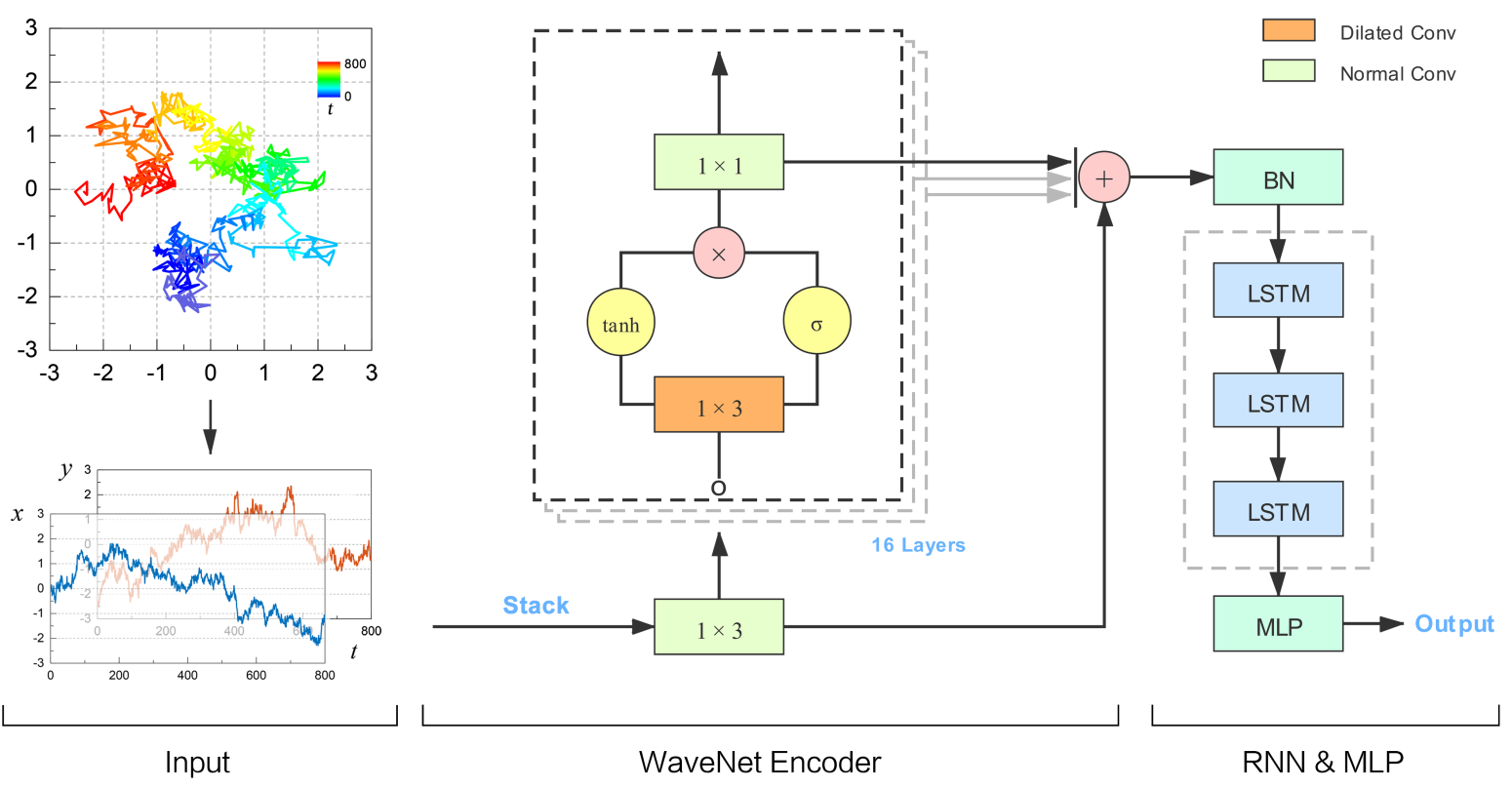

Figure 2. Overview of the workflow of WADNet. The workflow of WADNet consists of three main parts: Input, WaveNet Encoder, and RNN \& MLP. The Input part describes the preprocessing method of raw trajectories. The WaveNet Encoder part shows the detailed architecture of the modified WaveNet encoder. The output of this encoder is utilized as the input of the following $R N N \& M L P$ part, in which the inference and classification tasks are accomplished.

structures, named as "filter" and "gate". The outputs of "filter" and "gate" are activated by the hyperbolic tangent and sigmoid functions respectively, leading an output $\mathbf{z}_{k}$ of the gated activation unit:

$$
\mathbf{z}_{k}=\tanh \left(W_{f, k} * \tilde{\mathbf{x}}_{k}\right) \otimes \sigma\left(W_{g, k} * \tilde{\mathbf{x}}_{k}\right) .
$$

Here, $k$ is the layer index, $\tilde{\mathbf{x}}_{k}$ is the input tensor of the $k$-th layer, $W$ represents the dilated convolution filter with filter size $1 \times 3$ and filter number $32, f$ and $g$ denote the "filter" and "gate", * denotes the convolution operator, $\tanh (\cdot)$ is the hyperbolic tangent activation function, $\sigma(\cdot)$ is the sigmoid activation function, $\otimes$ denotes the element-wise multiplication operator. After that, a $1 \times 1$ convolution filter $W_{1}$ with filter number 32 is applied on $\mathbf{z}_{k}$ to obtain the input of next layer, written as:

$$
\tilde{\mathbf{x}}_{k+1}=W_{1} * \mathbf{z}_{k}, \quad k=1,2,3, \cdots, d .
$$

In particular, this operation is different from that in the original WaveNet encoder, where residual [35] is used in the layer, i.e., $\tilde{\mathbf{x}}_{k+1}=W_{1} * \mathbf{z}_{k}+\tilde{\mathbf{x}}_{k}$. The output tensor $\mathbf{u}$ of this modified encoder is obtained through skip connections:

$$
\mathbf{u}=\mathrm{BN}\left(\sum_{k=1}^{d+1} \tilde{\mathbf{x}}_{k}\right)
$$

where $\mathrm{BN}(\cdot)$ represents the $1 \mathrm{D}$ batch normalization.

At last, $\mathbf{u}$ is used as the input of the following RNN module. As show in the $R N N$ \& MLP part of Figure 2, this module is made up of 3 stacked LSTM units, whose 
dimension of hidden layers is 64 . The output $\mathbf{v}$ of RNN module is the feature vector of original trajectory learned by WADNet. To accomplish the challenge task, feature $\mathbf{v}$ is processed by a multilayer perceptron (MLP) network. For the inference task, the output dimension of MLP is 1 and no activation function is utilized; for the classification task, the output dimension of MLP is 5 and the activation function is the softmax function.

\subsection{Training procedure and inference strategy}

Models for each specific length are trained separately. $80 \%$ of training data is used for training, while the other is used for validation. The model is trained using backpropagation with a batch size 512, where loss function is the mean squared error (MSE) and cross entropy $(\mathrm{CE})$ for the inference and classification tasks, respectively. The optimizer is Adam with an initial learning rate $l=0.001$. During the training process, the learning rate is changed as $l_{\text {new }}=l_{\text {old }} / 5$ if the valid loss doesn't decrease after 2 epochs. When the number of such learning-rate changes exceeds 2 , the training process is early stopped to save training time and avoid overfitting.

To demonstrate the convergence of our method, we take the 1D trajectory at length 300 as the example, and show corresponding evolutions of train loss, valid loss, and valid metric in Figures 3a (the inference task) and 3b (the classification task). Since train and valid losses can both be effectively reduced during training, an excellent convergence of WADNet can be identified. In particular, we determine the best model weight for a specific length by the best valid metric, as depicted by the arrows in Figures 3a and 3b. Moreover, to examine the model performance in the training process, we show the best valid metrics of all specific lengths in all dimensions in Figures 3c (the inference task) and 3d (the classification task). For both two tasks, the performance of WADNet is better for higher spatial dimensions, and is gradually boosted as the trajectory length increases. This result arises from the fact that a longer trajectory with a higher dimension can provide more data points and hidden information.

Next, we pay attention to the inference of test dataset. Note that the length of a trajectory in the test dataset varies from 10 to 999 points. Since we only have model weights for 43 specific lengths, our model cannot be directly applied for inference. To address this issue, the inference of test dataset is guided by the following strategy:

- If the original length of a trajectory belongs to these 43 specific lengths, the data of this trajectory will be directly used for inference.

- Otherwise, a new length of this trajectory will be set as the closest smaller specific length. For instance, the new length of a trajectory with an original length 49 should be 45 . The trajectory data is subsequently transformed into 2 sequences. For clarity, we set the trajectory as $\mathbf{x}=\left[x_{1}, x_{2}, \cdots, x_{T}\right]$, where $T$ is the original length. We denote $T_{n}$ as the new length. Note that $T_{n}<T$, two sequences $\mathbf{x}_{1}=\left[x_{1}, x_{2}, \cdots, x_{T_{n}}\right]$ and $\mathbf{x}_{2}=\left[x_{T-T_{n}+1}, x_{T-T_{n}+2}, \cdots, x_{T}\right]$ with length $T_{n}$ can be obtained. Such two sequences are both used for inference, where corresponding feature vectors learned by WADNet are $\mathbf{v}_{1}$ and $\mathbf{v}_{2}$ respectively. After that, we 

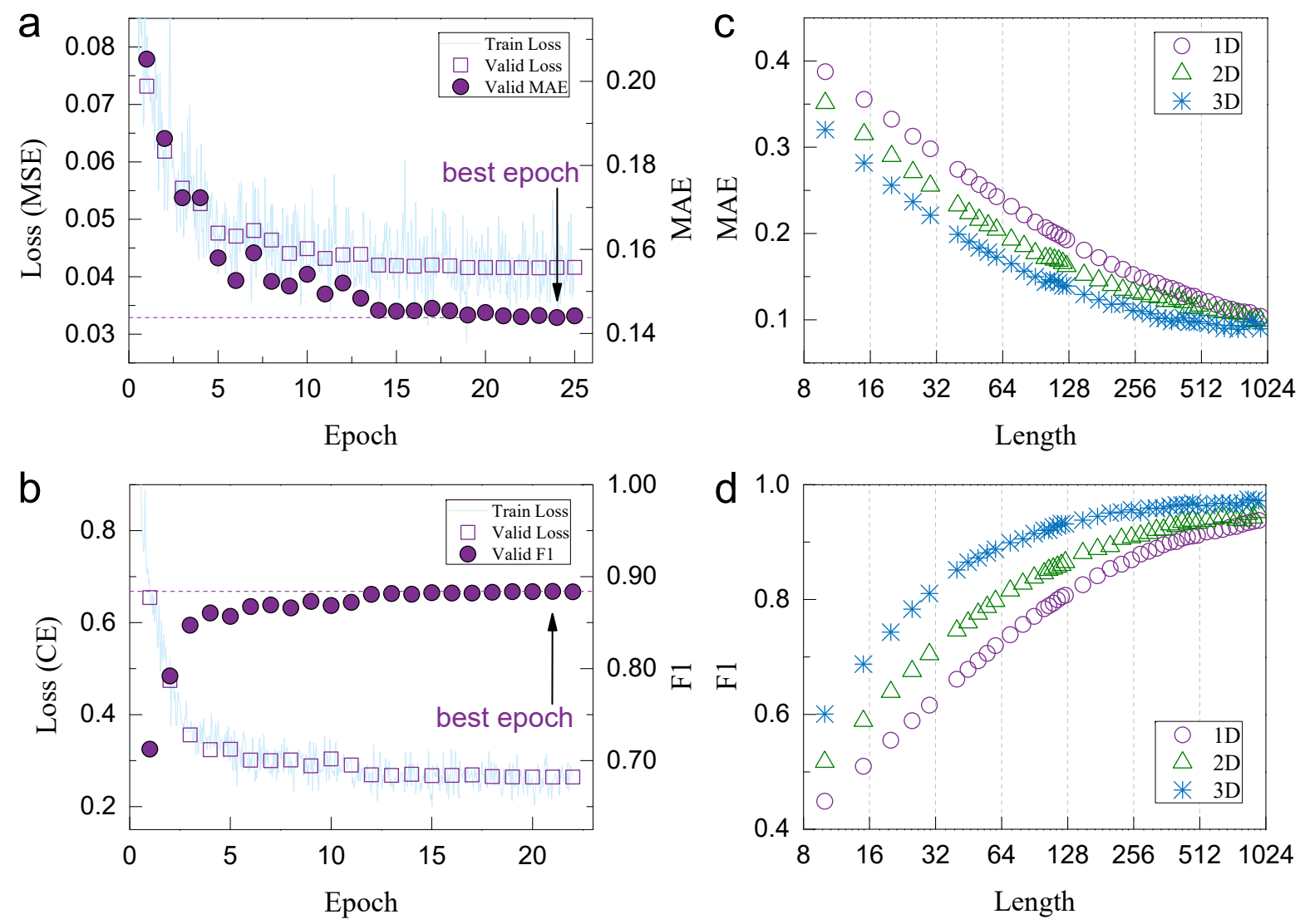

Figure 3. Model performance in the training process. (a-b) Evolutions of train loss, valid loss, and valid metric for the $1 \mathrm{D}$ trajectory at length 300 , regarding the inference task (a) and the classification task (b). The arrow denotes the best model weight for a specific length, which is determined by the best valid metric. (c-d) The best valid metrics of all specific lengths in all dimensions for the inference task (c) and the classification task (d).

choose the mean vector of these two vectors as the approximation of the feature $\mathbf{v}$ of the original trajectory, i.e., $\mathbf{v} \approx\left(\mathbf{v}_{1}+\mathbf{v}_{2}\right) / 2$. The final prediction is made based on $\mathbf{v}$ by the MLP.

The codes of WADNet for training and inference are freely available online [55], where the neural networks are implemented by PyTorch 1.6.0.

In addition, to further improve the performance of WADNet, $K$-fold cross validation technique $[56$ is utilized for both two tasks. In detail, the training dataset is randomly split into $K$ folds where $K=5$ in this work. When training a model, one fold is utilized as the validation data while other folds are used as training data. Such process is then repeated $K$ times, with each of the folds used exactly once as the validation data. Thus, the prediction of a sample can be obtained by averaging the results of $K$ single-fold models. On the other hand, for the inference of the anomalous diffusion exponent, predictions of test dataset are all rectified by multiplying a constant $w$ and are subsequently clipped to ensure $\alpha \in[0.05,2.0]$. The constant $w$ is determined by 

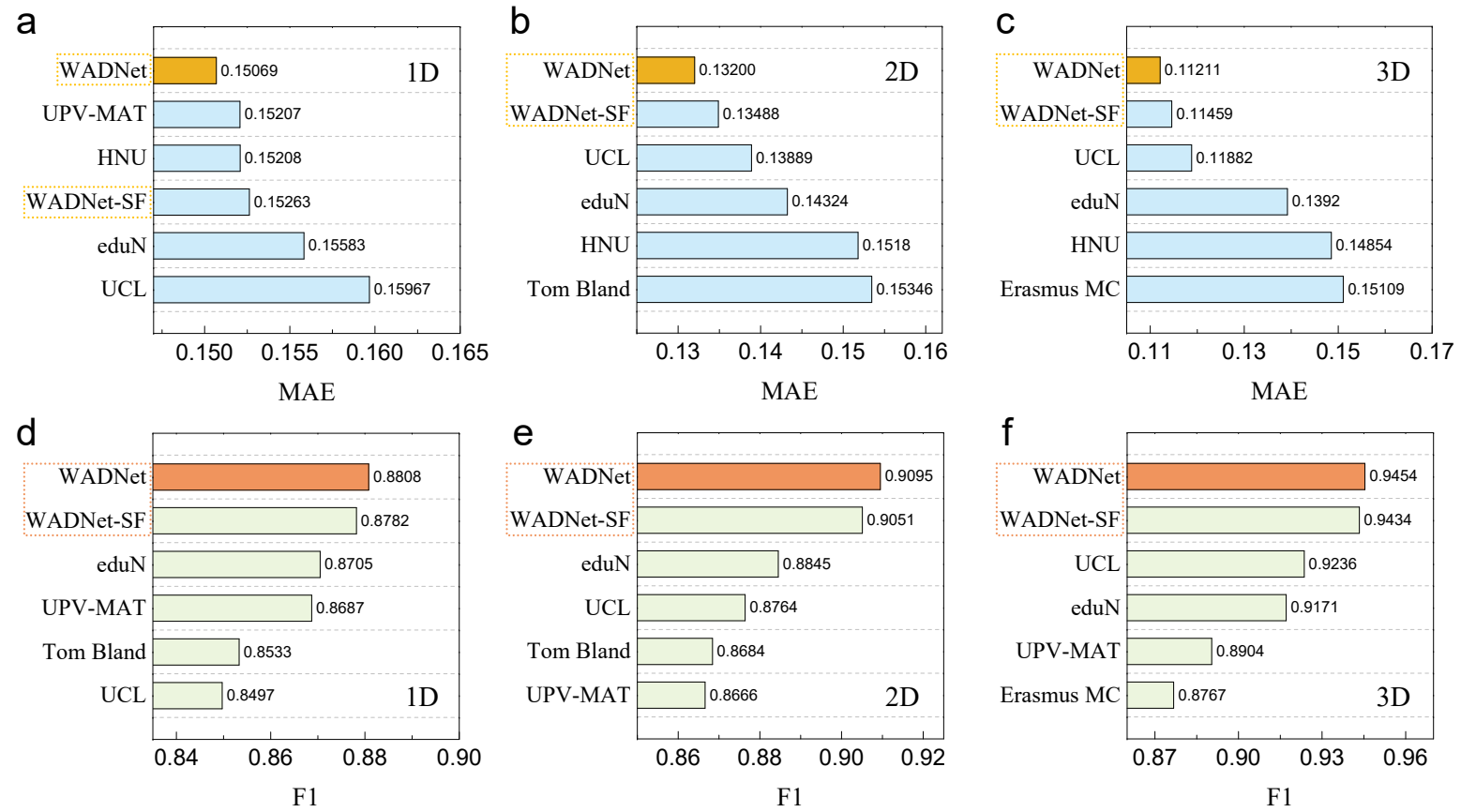

Figure 4. Performance of WADNet in the challenge leaderboard. Results of comparisons between WADNet and the top 4 ranks in the challenge leaderboard. Label "WADNet" denotes the score of our method improved by the $K$-fold cross validation, and label "WADNet-SF" denotes the score of a single-fold WADNet model. The best score in each subtask is marked in different color for clarity. (a-c) MAE scores in the inference task for 1D (a), 2D (b), and 3D (c) trajectories. (d-f) F1-scores in the classification task for 1D (d), 2D (e), and 3D (f) trajectories.

analyzing an external validation dataset containing 100000 trajectories, and is identified to be 1.0110, 1.0129, and 1.0083 for 1D, 2D, and 3D trajectories, respectively.

\section{Results and Discussions}

\subsection{Performance of WADNet in the challenge leaderboard}

To examine objectively the performance of WADNet in the AnDi Challenge, we compare our scores of test dataset with the top 4 ranks in the challenge leaderboard. The results of comparisons for both the inference and classification tasks in all dimensions (6 subtasks) are summarized in Figure 4, where the best scores are marked in different colors for clarity. It can be found that the scores of our method have surpassed the current 1st places in the leaderboard in all 6 subtasks. In particular, without the improvement of $K$-fold cross validation, the scores of a single-fold model (WADNet$\mathrm{SF}$ ) have also surpassed the 1st places in 5 subtasks. This result illustrates that the ability of WADNet in the characterization of anomalous diffusion mainly arises from the model itself. Therefore, WADNet could be the part of state-of-the-art techniques for the AnDi database. Note that there are only 43 specific lengths in the training dataset. That is, by a simple expansion of training data, the performance of WADNet can be 



Figure 5. Differences between the predicted values and ground truth values of the anomalous diffusion exponent. (a-c) 2D histograms of $\alpha_{\mathrm{p}}$ versus $\alpha_{\mathrm{GT}}$ for 1D (a), 2D (b), and 3D (c) trajectories. (d-f) Plots of MAE versus $\alpha_{\mathrm{GT}}$ for 1D (d), 2D (e), and 3D (f) trajectories. The dashed lines denote the mean values of MAE.

further improved. Analysis in the following sections is based on the best performance of WADNet that is improved by the $K$-fold cross validation.

\subsection{Analysis of the inference of the anomalous diffusion exponent}

In this section, we provide a detailed analysis of the performance of WADNet on the inference task. Firstly, as the spatial dimension of trajectory increases, MAE value of test dataset is reduced from 0.15069 (1D) to 0.13200 (2D) to 0.11211 (3D). This result can be attributed to the extra information given by a higher dimension. Moreover, to intuitively demonstrate the differences between the predicted values $\alpha_{\mathrm{p}}$ and ground truth values $\alpha_{\mathrm{GT}}$, we plot the 2D histograms of $\alpha_{\mathrm{p}}$ versus $\alpha_{\mathrm{GT}}$ for all dimensions in Figures 5a-c. It can be found that $1 \mathrm{D}$ and $2 \mathrm{D}$ trajectories with $\alpha_{\mathrm{GT}} \approx 1.0$ are more likely to be predicted as subdiffusion $\left(\alpha_{\mathrm{p}}<1\right)$, while such a deviation is slightly eliminated in $3 \mathrm{D}$ space. This bias can also be captured by the plots of MAE versus $\alpha_{\mathrm{GT}}$ (Figures $5 \mathrm{~d}-\mathrm{f})$, where MAE values at $\alpha_{\mathrm{GT}} \approx 1.0$ are relatively higher than that of neighborhoods. Meanwhile, significant errors can be identified at $\alpha_{\mathrm{GT}} \rightarrow 0$. The reason is that a random walker in this case is nearly motionless and corresponding trajectory is more like a record of localization noise.

Next, we explore the dependence of model performance on different diffusion models, and show the results in Figure 6a. As expected, FBM that is ergodic has the lowest MAE values in all dimensions. Interestingly, for those non-ergodic models, 

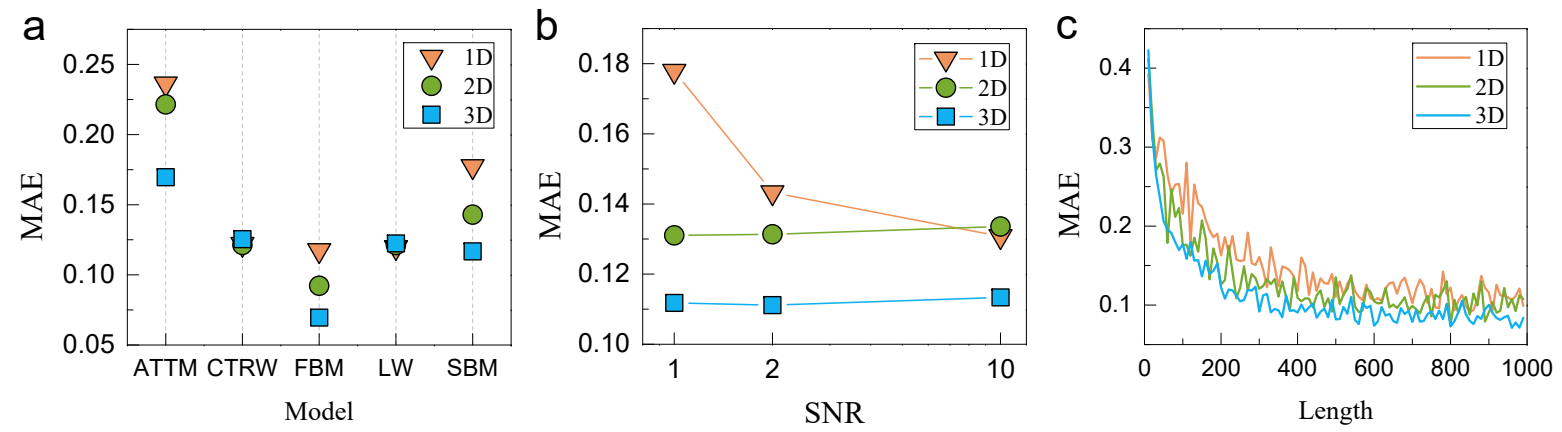

Figure 6. Influence of the diffusion model, SNR, and trajectory length on the inference task. (a) MAE of 5 diffusion models. (b) Plots of MAE versus SNR. (c) Dependence of MAE on the trajectory length.

WADNet shows a better performance on CTRW and LW than on ATTM and SBM, indicating that trajectories of CTRW and LW could provide more hidden information than the other two. Additionally, MAE values of CTRW and LW are nearly independent of the trajectory dimension, while those of ATTM, FBM, and SBM decrease as the dimension increases. This result suggests that the quality of features of CTRW and LW learned by WADNet is less correlated with the spatial dimension.

On the other hand, since experimental trajectories can usually be noisy, it is of crucial importance to examine the performance of WADNet on noisy trajectories. For that purpose, we investigate the effects of SNR on the performance of our model. As shown in Figure 6b, MAE value can be slightly reduced by the improvement of SNR for the $1 \mathrm{D}$ trajectory. However, a surprising result is that MAE values are nearly the same for all SNR in 2D and 3D spaces. Such a result implies that WADNet has the ability to remove the undesirable effects introduced by localization noise for $2 \mathrm{D}$ and $3 \mathrm{D}$ trajectories, even for trajectories where the standard deviation of noise has the same amplitude as that of displacement (i.e., SNR =1). Therefore, our method is of high potential to be utilized for the analysis of noisy experimental trajectory data. At last, the influence of trajectory length is demonstrated in Figure 6c. As expected, MAE rapidly decreases with the increase of trajectory length, and reaches a relatively steady value near the length 600 .

\subsection{Analysis of the classification of the diffusion model}

In this section, we focus attention on the performance of WADNet on the classification task. At first, F1-score of test dataset is boosted from 0.8808 (1D) to 0.9095 (2D) to $0.9454(3 \mathrm{D})$, as a consequence of the information gain given by a higher spatial dimension. To examine the classification performance of WADNet in detail, we show the confusion matrixes for 1D, 2D and 3D trajectories in Figures 7a-c respectively. For clarity, we use the percentage instead of sample number as the element of confusion matrix. Thus, the diagonal element of matrix is actually the classification accuracy of WADNet for each model. As characterized by the accuracy, the performance on CTRW 

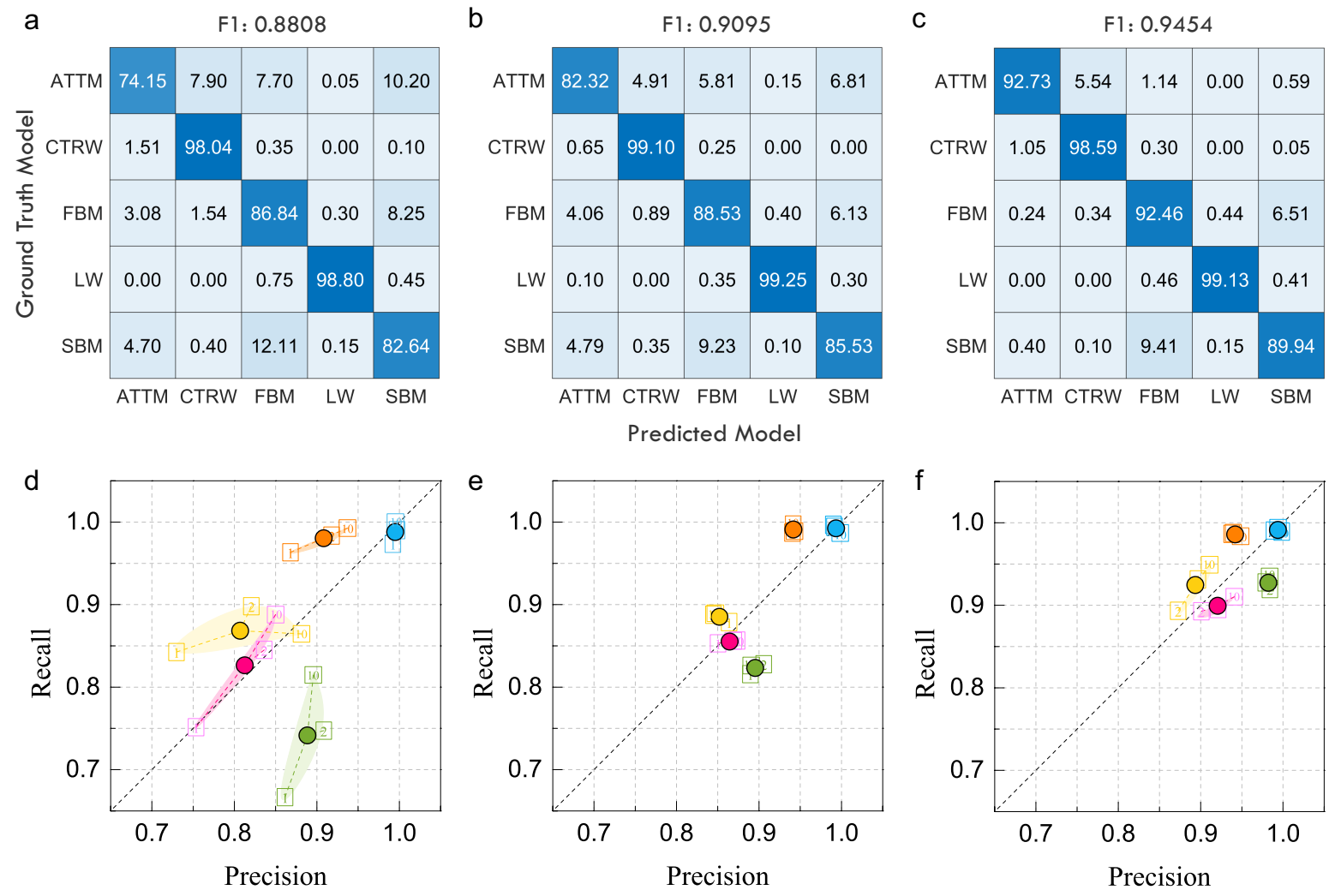

○ ATTM $\bigcirc$ CTRW $\bigcirc$ FBM $\bigcirc \quad$ LW $\bigcirc \quad$ SBM

Figure 7. Detailed analysis of the performance of WADNet on the classification task. (a-c) Confusion matrixes of classification results for 1D (a), 2D (b) and 3D (c) trajectories. The element of confusion matrix is the percentage, and the diagonal element of matrix is the classification accuracy for each model. (d-f) Plots of precision versus recall for 1D (d), 2D (e) and 3D (f) trajectories. The dots denote the overall scores, and the squares denote the results for different SNR. The colored region is a guide of square locations to the eye, and its area reports the stability of WADNet on noisy trajectories for each model.

and LW is relatively better (accuracy $>98 \%$ in all dimensions) and almost independent on the spatial dimension, while the performance on ATTM, FBM, and SBM is gradually improved as the dimension increases.

From another perspective, the classification ability of WADNet is further analyzed by calculating the precision and recall scores of each diffusion model. The plots of precision versus recall for all dimensions are given in Figures $7 \mathrm{~d}-\mathrm{f}$ as dots, where the diffusion models are distinguished by different colors. A general phenomenon in all dimensions is that precision and recall are approximately equivalent for LW and SBM, but show non-negligible differences for the other 3 models. In more detail, the recall is smaller than precision for ATTM, and undergoes a reverse for FBM and CTRW. That is, regarding the error of classifying a trajectory, ATTM is more likely to be mistaken, while FBM and CTRW are more preferred to be the targets.

Next, similar with the inference task, the performance of WADNet on noisy 

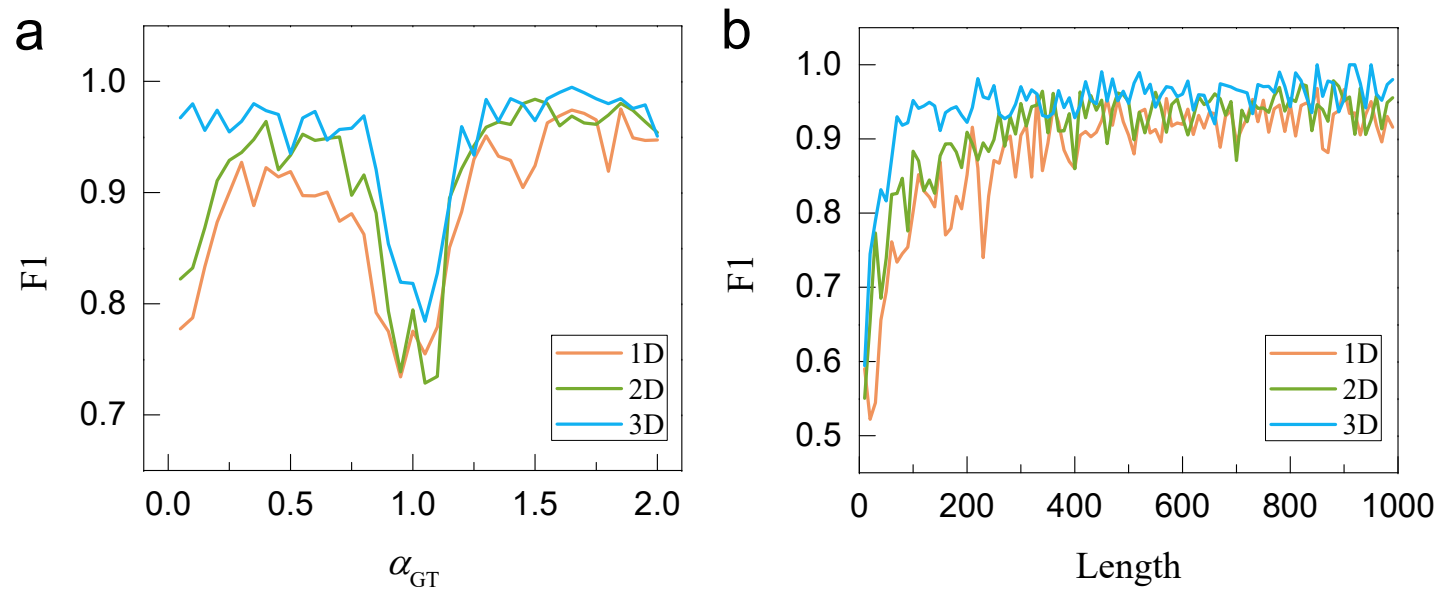

Figure 8. Influence of anomalous diffusion exponent and trajectory length on the classification task. (a) Plots of F1-score as a function of $\alpha_{\mathrm{GT}}$. (b) Plots of F1-score versus trajectory length.

trajectories is also examined by studying the effects of SNR. Corresponding results in all dimensions are plotted in Figures $7 \mathrm{~d}-\mathrm{f}$ as squares marked by SNR values. The colored region is a guide of square locations to the eye, and its area reports the stability of our method on noisy trajectories for each model. As characterized by the small area of colored regions, insensitivity of F1-score to SNR can be identified for LW and CTRW in all dimensions. In contrast, F1-scores of ATTM, FBM, and SBM in 1D space can be slightly improved by the increase of SNR. However, for 2D and 3D trajectories, F1-scores of these 3 models are nearly independent of SNR, as the same with LW and CTRW. Thus, negative influences of localization noise on the classification performance could be vastly eliminated by WADNet.

Moreover, to investigate the influence of anomalous diffusion exponent on the classification result, we show the F1-score as a function of $\alpha_{\mathrm{GT}}$ in Figure 8a. Obviously, the worst performance of WADNet can be identified for trajectories with $\alpha_{\mathrm{GT}} \approx 1.0$ in all dimensions. The possible reason is that the dynamics of these trajectories is close to Brownian motion, which leads to similar statistical properties regardless of the specific diffusion model. In addition, the classification performance is also poor at $\alpha_{\mathrm{GT}} \rightarrow 0$ in $1 \mathrm{D}$ and 2D spaces. This result can be attributed to the fact that the transport of a random walker with an extremely small $\alpha$ is nearly immobile and dominated by the random noise. This phenomenon vanishes for $3 \mathrm{D}$ trajectories, probably owing to the information gain. In addition, the dependence of F1-score on trajectory length is given in Figure 8b. As was anticipated, F1-score undergoes a rapid growth as the trajectory length increases, and reaches a relatively steady value near the length 600 .

At last, to gain a more in-depth insight into the classification ability of our method, we present a visualization of feature vector $\mathbf{v}$ extracted from the penultimate layer of WADNet. Figure 9 shows the uniform manifold approximation and projection for dimension reduction (UMAP) [57 projections of feature vectors of $1 \mathrm{D}$ trajectories with 


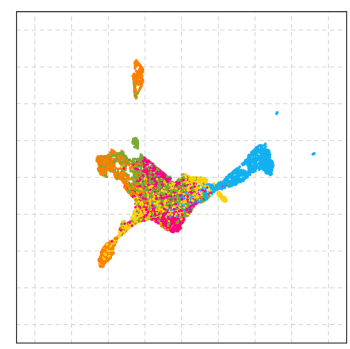

Length $=40$



Length $=200$

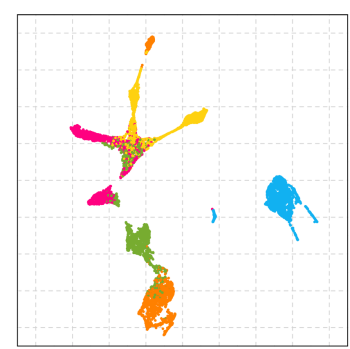

Length $=400$

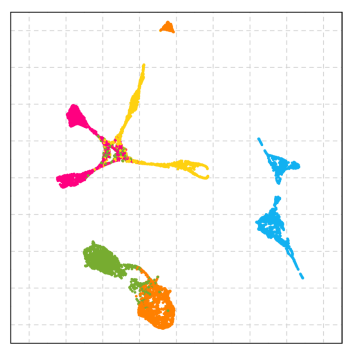

Length $=900$

- ATTM $\odot$ CTRW $\odot$ FBM $\odot \mathrm{LW} \odot \mathrm{SBM}$

Figure 9. Visualization of learned features. Uniform manifold approximation and projection for dimension reduction (UMAP) projections of feature vectors of $1 \mathrm{D}$ trajectories with length 40,200,400, and 900, where each length has 10000 trajectories. Feature vectors are extracted from the penultimate layer of WADNet.

length 40, 200, 400, and 900, where each length has 10000 trajectories. Projections of features are marked by different colors according to corresponding diffusion models. It can be found that features are mixed for short trajectories, but can be clearly distinguished for long trajectories. In more detail, as the trajectory length increases, the features of LW can be firstly separated (length 200), and two main isolated clusters can be identified next (length 400 and 900). One cluster is primarily composed of FBM and SBM, and the other is a combination of CTRW and ATTM. Therefore, from the perspective of feature similarity, the underlying dynamics of these diffusion models is not completely uncorrelated. The overlap between FBM and SBM suggests that the diffusion driven by a fractional Gaussian noise can sometimes be similar with the Brownian motion with a time-dependent diffusivity. Meanwhile, the effects of an irregular waiting time might be approximated by a random variation of diffusivity at times, as indicated by the adjacency between CTRW and ATTM. For future research, these features learned by WADNet have the potential to be used as quantitative representations of trajectories with multiple and mixed diffusion dynamics.

\section{Conclusions}

In conclusion, by combining a modified WaveNet encoder with LSTM networks, we have developed a WaveNet-based deep neural network (WADNet) for the characterization of anomalous diffusion in response to the AnDi Challenge. Without any prior knowledge of anomalous diffusion, the performance of WADNet has surpassed the current 1st places in the challenge leaderboard for all dimensions, with respect to the inference of the anomalous diffusion exponent and the classification of the diffusion model. That is, WADNet could be the part of state-of-the-art techniques to decode the AnDi database. Moreover, the results show that WADNet can effectively extract information from noisy trajectories and vastly eliminate the undesirable influences of localization noise. The features learned by WADNet have the potential to be utilized as the quantitative 
representation of complex anomalous diffusion dynamics. In particular, the main architectures of WADNet for different tasks are nearly the same, where modifications are only applied on the input and output dimensions of network. Thus, our findings bring new insights into the generalization of anomalous diffusion, and could accelerate the design of a versatile strategy for single trajectory characterization.

\section{Acknowledgements}

We thank the organizers of the AnDi Challenge for providing the database of simulated trajectories. This work is supported by the Fundamental Research Funds for the Central Universities.

\section{References}

[1] Metzler R, Jeon J-H, Cherstvy A G and Barkai E 2014 Anomalous diffusion models and their properties: non-stationarity, non-ergodicity, and ageing at the centenary of single particle tracking Phys. Chem. Chem. Phys. 16 24128-64

[2] Klafter J and Sokolov I M 2005 Anomalous diffusion spreads its wings Phys. World 1829

[3] Mason T G and Weitz D A 1995 Optical measurements of frequency-dependent linear viscoelastic moduli of complex fluids Phys. Rev. Lett. 741250

[4] Aarão Reis F D A 2016 Scaling relations in the diffusive infiltration in fractals Phys. Rev. E 94 052124

[5] Volpe G and Volpe G 2017 The topography of the environment alters the optimal search strategy for active particles Proc. Natl. Acad. Sci. USA 114 11350-5

[6] Xu Z, Dai X, Bu X, Yang Y, Zhang X, Man X, Zhang X, Doi M and Yan L-T 2021 Enhanced heterogeneous diffusion of nanoparticle in semiflexible networks ACS Nano 15 4608-16

[7] Barkai E, Garini Y and Metzler R 2012 Strange kinetics of single molecules in living cells Phys. Today 6529

[8] Höfling F and Franosch T 2013 Anomalous transport in the crowded world of biological cell Rep. Prog. Phys. 76046602

[9] Wu X-L and Libchaber A 2000 Particle diffusion in a quasi-two-dimensional bacterial bath Phys. Rev. Lett. 843017

[10] González M C, Hidalgo C A and Barabási A-L 2008 Understanding individual human mobility patterns Nature 453 779-82

[11] Wang B, Kuo J and Granick S 2013 Bursts of active transport in living cells Phys. Rev. Lett. 111 208102

[12] Chen P, Huang Z, Liang J, Cui T, Zhang X, Miao B and Yan L-T 2016 Diffusion and directionality of charged nanoparticles on lipid bilayer membrane ACS Nano 10 11541-7

[13] Plerou V, Gopikrishnan P, Amaral L A N, Gabaix X and Stanley H E 2000 Economic fluctuations and anomalous diffusion Phys. Rev. E 62 R3023

[14] Masoliver J, Montero M and Weiss G H 2003 Continuous-time random-walk model for financial distributions Phys. Rev. E 67021112

[15] Jiang Z-Q, Xie W-J, Zhou W-X and Sornette D 2019 Multifractal analysis of financial markets: a review Rep. Prog. Phys. 82125901

[16] Castellano C, Fortunato S and Loreto V 2009 Statistical physics of social dynamics Rev. Mod. Phys. $\mathbf{8 1} 591$

[17] Manzo C and Garcia-Parajo M F 2015 A review of propress in single particle tracking: form methods to biophysical insights Rep. Prog. Phys. 78124601 
[18] Shen H, Tauzin L J, Baiyasi R, Wang W, Moringo N, Shuang B and Landes C F 2017 Single particle tracking: from theory to biophysical applications Chem. Rev. 117 7331-76

[19] Qian H, Sheetz M P and Elson E L 1991 Single particle tracking. analysis of diffusion and flow in two-dimensional systems Biophys. J. 60 910-21

[20] Saxton M J 2008 Single-particle tracking: connecting the dots Nat. Meth. 5 671-2

[21] Torreno-Pina J A, Manzo C and Garcia-Parajo M F 2016 Uncovering homo-and hetero-interactions on the cell menbrane using single particle tracking approaches J. Phys. D: Appl. Phys. 49104002

[22] Wong I Y, Gardel M L, Reichman D R, Weeks E R, Valentine M T, Bausch A R and Weitz D A 2004 Anomalous diffusion probes microstructure dynamics of entangled F-actin networks Phys. Rev. Lett. 92178101

[23] Banks D S and Fradin C 2005 Anomalous diffusion of proteins due to molecular crowding Biophys. J. 89 2960-71

[24] Chen D T N, Lau A W C, Lslam M F, Goulian M, Lubensky T C and Yodh A G 2007 Fluctuations and rheology in active bacterial suspensions Phys. Rev. Lett. 99148302

[25] Huang Z, Chen P, Zhu G, Yang Y, Xu Z and Yan L-T 2018 Bacteria-activated Janus particles driven by chemotaxis ACS Nano $126725-33$

[26] Chen P, Yue H, Zhai X, Huang Z, Ma G-H, Wei W and Yan L-T 2019 Transport of a graphene nanosheet sandwiched inside cell membranes Sci. Adv. 53192

[27] Lozano C, Gomez-Solano J R and Bechinger C 2019 Active particles sense micromechanical properties of glasses Nat. Mater. 18 1118-23

[28] Wang B, Anthony S M, Bae S C and Granick S 2009 Anomalous yet Brownian Proc. Natl. Acad. Sci. USA 106 15160-4

[29] Kim J, Kim C and Sung B J 2013 Simulation study of seemingly Fickian but heterogeneous dynamics of two dimensional colloids Phys. Rev. Lett. 110047801

[30] Weron A, Janczura J, Boryczka E, Sungkaworn T and Calebiro D 2019 Statistical testing approach for fractional anomalous diffusion classification Phys. Rev. E 99042149

[31] Jeon J-H and Metzler R 2010 Analysis of short subdiffusive time series: scatter of the time-avaraged mean-squared displacement J. Phys. A: Math. Theor. 43252001

[32] Akin E J, Solé L, Johnson B, el Beheiry M, Masson J-B, Krapf D and Tamkun M M 2016 Singlemolecule imaging of $\mathrm{Na}_{\mathrm{v}} 1.6$ on the surface of hippocampal neurons reveals somatic nanoclusters Biophys. J. 111 1235-47

[33] Muñoz-Gil G, Volpe G, Garcia-March M A, Metzler R, Lewenstein M and Manzo C 2020 AnDi: the anomalous diffusion challenge (arXiv:2003.12036)

[34] Silver D et al 2016 Mastering the game of Go with deep neural networks and tree search Nature 529 484-9

[35] He K, Zhang X, Ren S and Sun J 2016 Deep residual learning for image recognition Proc. of the IEEE Conf. on Computer Vision and Pattern Recognition pp 770-8

[36] Hochreiter S and Schmidhuber J 1997 Long short-term memory Neural Comput. 9 1735-80

[37] Cho K, van Merriënboer B, Gulcehre C, Bahdanau D, Bougres F, Schwenk H and Bengio Y 2014 Learning phrase representations using RNN encoder-decoder for statistical machine translation Proc. of the Conf. on Empirical Methods in Natural Language Processing pp 1724-34

[38] van den Oord A, Dieleman S, Zen H, Simonyan K, Vinyals O, Graves A, Kalchbrenner N, Senior A and Kavukcuoglu K 2016 WaveNet: a generative model for raw audio (arXiv:1609.03499)

[39] Vaswani A, Shazeer N, Parmar N, Uszkoreit J, Jones L, Gomez A N, Kaiser L and Polosukhin I 2017 Attention is all you need Proc. of the Int. Conf. on Neural Information Processing Systems pp 6000-10

[40] Bo S, Schmidt F, Eichhorn R and Volpe G 2019 Measurement of anomalous diffusion using recurrent neural networks Phys. Rev. E $100010102(\mathrm{R})$

[41] Muñoz-Gil G, Garcia-March M A, Manzo C, Marthín-Guerrero J D and Lewenstein M 2020 Single trajectory characterization via machine learning New J. Phys. 22013010

[42] Muñoz-Gil G et al 2021 Objective comparison of methods to decode anomalous diffusion 
(arXiv:2105.06766)

[43] Gentili A and Volpe G 2021 Characterization of anomalous diffusion classical statistics powered by deep learning (CONDOR) (arXiv:2102.07605)

[44] Verdier H, Duval M, Laurent F, Cassé A, Vestergaard C and Masson J-B 2021 Learning physical properties of anomalous random walks using graph neural networks (arXiv:2103.11738)

[45] Argun A, Volpe G and Bo S 2021 Classification, inference and segmentation of anomalous diffusion with recurrent neural networks (arXiv:2104.00553)

[46] Manzo C 2021 Extreme learning machine for the characterization of anomalous diffusion from single trajectories (arXiv:2105.02597)

[47] Massignan P, Manzo C, Torreno-Pina J A, García-Parajo M F, Lewenstein M and Lapeyre Jr G J 2014 Nonergodic subdiffusion from Brownian motion in an inhomogeneous medium Phys. Rev. Lett. 112150603

[48] Scher H and Montroll E W 1975 Anomalous transit-time dispersion in amorphous solids Phys. Rev. B 122455

[49] Mandelbrot B B and Ness J W V 2006 Fractional Brownian motions, fractional noises and applications SIAM Rev. 10 422-37

[50] Klafter J and Zumofen G 1994 Lévy statistics in a Hamiltonian system Phys. Rev. E 494873

[51] Lim S C and Muniandy S V 2002 Self-similar Gaussian processes for modeling anomalous diffusion Phys. Rev. E 66021114

[52] Muñoz-Gil G, Manzo C, Volpe G, Garcia-March M A, Metzler R and Lewenstein M 2020 The anomalous diffusion challenge dataset URL https://zenodo.org/record/3707702

[53] Kleijn W B, Lim F S C, Luebs A, Skoglund J, Stimberg F, Wang Q and Walters T C 2018 Wavenet based low rate speech coding Proc. of the IEEE Int. Conf. on Acoustics, Speech and Signal Processing pp 676-80

[54] Chorowski J, Weiss R J, Bengio S and van den Oord A 2019 Unsupervised speech representation learning using WaveNet autoencoders IEEE ACM Trans. Audio Speech Lang. Process. 27 204153

[55] Li D, Yao Q and Huang Z 2021 WADNet URL https://github.com/huangzih/WADNet

[56] Stone M 1974 Cross-validatory choice and assessment of statistical predictions J. Roy. Stat. Soc. B 36 111-47

[57] Mclnnes L, Healy J and Melville J 2020 UMAP: uniform manifold approximation and projection for dimension reduction (arXiv:1802.03426) 